Meddelelser fra Dansk Naturhistorik Forening i Kjobenhavn 2: 297-314.

MOEBIUS, M. -1889- Bearbeitung der von $\mathrm{H}$. Schenck in Brasilien gesammelten Algen. Hedwigia 28(5): 309-347.

MOEBIUS, M. -1890- Algae brasiliensis a Dr. Glaziou collectae. Notarisia 5(20): 1065-1090. TAYLOR, W.R. -1960- Marine algae of Eastern tropical and subtropical coasts of the Americas. The University of Michigan Press.

VASQUES MARTINS, D., M. CORDEIROMARINO, N. BARBOSA BOCCANERA y J.M. CASTRO NUNES - 1991-Clorofíceas marinhas bentônicas do Munícipio de Salvador, Bahia, Brasil. Hoehnea 18(2): 115-133.
WYNNE, M.J. - 1986- A check-list of benthic marine algae of the tropical and subtropical western Atlantic. Canadian Journal of Botany 64: 22392281 .

Aceptado para su publicación en Mayo de 1997

Dirección de los autores. M. ALTAMIRANO: Departamento de Biología Vegetal. Facultad de Ciencias. Universidad de Málaga. Campus de Teatinos s/n. E-29071 Málaga. España. J.M. de CASTRO NUNES: Departamento de Botânica, Instituto de Biologia da Universidade Federal da Bahia. Campus Universitario de Ondina. CEP 40.170-210, Salvador, Bahia, Brasil.

\title{
60. REFERENCIAS COROLÓGICAS DE PLANTAS VASCULARES PARA EL SE. IBÉRICO
}

\author{
Arturo VALDÉS FRANZI y Rafael MOLINA CANTOS
}

Geographical distribution references of vascular plants for Southeastern Spain.

Palabras clave. Plantas vasculares, corología, SE. España.

Key words. Vascular plants, geographical distribution, Southeastern Spain.

Se ofrece una relación de plantas vasculares recolectadas en su mayoría en el $\mathrm{N}$. de Albacete, España. Las especies señaladas con un asterisco constituyen novedades de ámbito provincial. El resto son segundas citas para la provincia de Albacete de plantas poco frecuentes que amplían el área conocida para dicha especie en más de $100 \mathrm{~km}$ y, en algún caso, confirman citas de autores antiguos.

Los taxones aparecen en orden alfabético. La nomenclatura y autorías de los mismos siguen a Flora Iberica (Castroviejo et al., 19861993) o, si no han sido publicados aún en dicha obra, a Flora Europaea (Tutin et al., 19641980).

Se conservan tusigos de todas las citas en el herbario ALBA de la Escuela Universitaria de Magisterio, Plaza de la Universidad, Campus Universitario. Albacete. España.

*Alnus glutinosa (L.) Gaertner CUENCA: Casas de Bénitez, La Losa, presa, 
WJ7759, 730 m, chopera ribereña, 12-VIII-1992, R. Molina \& E. López (ALBA 4595). Constituye una de las citas más meridionales para el SE. Ibérico y es probable su presencia en zonas próximas de la provincia de Albacete. Se confirma la cita genérica y con dudas de Castroviejo et al. (1990), para Cuenca.

Arabis sagittata (Bertol.) DC. in Lam. \& DC.

ALBACETE: Jorquera, XJ2837, $640 \mathrm{~m}$, olmeda, 8-V-1991, A. Valdés \& R. Molina (ALBA 3904). Alcalá del Júcar, XJ3433, $550 \mathrm{~m}$, olivar inculto con suelo húmedo, 2-VI-1989, R. Molina \& J.L. González (ALBA 3905). 2 $2^{\text {a }}$ cita provincial (Herranz \& Gómez Campo, 1986).

\section{Carex elata All. subsp. elata}

ALBACETE: Fuensanta, Puente de San Alejandro, WJ8242, $630 \mathrm{~m}$, sauceda semiinundada, 16-IV-1992, R. Molina (ALBA 4794). 2 $2^{\mathbf{a}}$ cita provincial después de la de Luceño (1994) en la que no se indica localidad concreta en la provincia.

\section{* Carex humilis Leysser}

ALBACETE: La Roda, Puente del Carrasco, WJ8440, 680 m, encinar, 6-III-1990, R. Molina (ALBA 2806). Jorquera, central hidroeléctrica, XJ2938, $620 \mathrm{~m}$, cantiles calizo-arcillosos, 3-III1989, R. Molina (ALBA 2807).

* Carex nigra (L.) Reichard

ALBACETE: Valdeganga, puente, XJ1433, $630 \mathrm{~m}$, terraplén semiinundado por el río Júcar, 19VI-1991, A. Valdés \& R. Molina (ALBA 2798). Alcalá del Júcar, Molino de Don Benito, XJ4340, $550 \mathrm{~m}$, en reculaje de presa sobre cantos rodados, 21-IV-1989, R. Molina \& J.L. González (ALBA 2799).

\section{Crepis pulchra L.}

ALBACETE: Valdeganga, Bolinches, XJI134, $620 \mathrm{~m}$, chopera sobre suelo embarrado en una represa, 19-VI-1991, A. VaLdés \& R. MoLina (ALBA 3775). Ampliación de área (Herranz \& Gómez Campo, 1986; Sánchez Gómez \& Alcaraz, 1993).

Crypsis aculeata (L.) Aiton

ALBACETE: Chinchilla, Hoya Husilla, XH2397, $860 \mathrm{~m}$, en formaciones de Scirpus maritimus sobre suelos húmedos, 3-X-1990, A. Valdés \& J.L. González (ALBA 1848). $2^{\text {a }}$ cita provincial (Rivas Martínez, 1966).

\section{* Chenopodium multifidum L.}

ALBACETE: Villalgordo del Júcar, puente, WJ8152, $670 \mathrm{~m}$, lindes de regadíos de maíz, 28-IX88, R. Molina (ALBA 2850). Albacete ciudad, Fábrica de harinas, WJ9918, $700 \mathrm{~m}$, solar abandonado, 8-IV-1996, E. Izquierdo \& A. Valdés (ALBA 2164). Naturalizada.

Ephedra distachya L. subsp. distachya ALBACETE: Villa de Ves, Cristo de la Vida, XJ5042, 740, fisuras de rocas calcáreas, 8-VI-1989, R. Molina \& J.L. González (ALBA 3040). Bormate, hacia Mahora, XJ1938, 670 m, ladera terrosopedregosa, 26-V-1992, R. MoLina (ALBA 5017). Fuensanta, hacia El Carrasco, WJ8242, $650 \mathrm{~m}$, paredón calizo, 16-IV-1992, R. Molina (ALBA 5018). $2^{\text {a }}$ cita provincial después de la genérica de Castroviejo et al. (1986).

\section{* Eryngium dichotomum Desf.}

ALBACETE: De Albacete hacia Motilleja, XJ0235, $680 \mathrm{~m}$, coscojar en ladera soleada, 20-VII1988, R. Molina (ALBA 3058).

\section{Ferula communis L.}

ALBACETE: Casas Ibáñez, hacia Alcalá del Júcar, XJ3347, $700 \mathrm{~m}$, cardunales en campos incultos, 27-V-1992, R. Molina (ALBA 5034). 2a cita provincial (Sánchez Gómez \& Alcaraz, 1993).

\section{Fumana laevis (Cav.) Pau}

ALBACETE: Casas de Juan Gil, Casa de la Pulga, XJ5347, $830 \mathrm{~m}$, encinar pastoreado, 21 -VI1992, R. Molina (ALBA 4846). 2a cita provincial (Moroder, 1936). También existe una cita genérica para la provincia en Castroviejo et al. (1993).

\section{* Hibiscus trionum L.}

ALBACETE: Alborea, hacia Casas Ibáñez, XJ3649, $640 \mathrm{~m}$, herbazal en cercanías de una acequia, 7-8-1992, R. Molina (ALBA 4622). Naturalizada.

Juncus effusus L. var. effusus y var subglomeratus DC. in Lam DC.

ALBACETE: Fuentealbilla, hacia Jorquera, 
XJ2445, $620 \mathrm{~m}$, juncales, 1-VI-1992, R. Molina (ALBA 5015). 2a cita provincial (Dantín Cereceda, 1911, como J. effusus).

\section{* Lappula squarrosa (Retz.) Dumort.}

ALBACETE: Albacete, encinar de Los Yesares, XJ0832, $690 \mathrm{~m}$, coscojar-romeral sobre rañas silíceas, 12-V-1989, R. Molina (ALBA 3083). Tarazona de la Mancha, La Marmota, WJ8737, 700 $\mathrm{m}$, en cardunales sobre suelo arenoso removido, 7VI-1992, R. Molina (ALBA 4633).

\section{Lemna gibba $\mathrm{L}$.}

ALBACETE: Alborea, hacia Casas Ibáñez, XJ3649, $640 \mathrm{~m}$, riachuelo con plantas flotantes, 7VIII-1992, R. Molina (ALBA 4634). 2a cita provincial (Herrero et al., 1994).

\section{* Linaria repens (L.) Miller}

(incl. L. blanca Pau)

ALBACETE: Carcelén, $2 \mathrm{~km}$ hacia Alatoz, XJ4429, $1020 \mathrm{~m}$, bosquete de encinas al pie de cantiles calizos, 11-VII-1992, A. Valdés \& R. Molina (ALBA 4638).

\section{* Orobanche rapum-genistae Thuill.}

ALBACETE: Alrededores de Villalgordo, hacia La losa, WJ7952, $670 \mathrm{~m}$, en bordes de caminos sobre Vicia faba, 1-V-1992, R. Molina (ALBA 4874). Hellín, Casas del Embalse, XH 1745, 450 m, romeral con Stipa tenacissima en laderas calcáreomargosas, 22-IV-1989, J.L. González (ALBA 4376).

\section{Parietaria lusitanica $\mathrm{L}$.}

ALBACETE: Alcalá del Júcar, Tolosa, XJ4039, $630 \mathrm{~m}$, terraplén calizo-margoso, 23-V1991, A. Valdés \& R. Molina (ALBA 3156). Villa de Ves, poblado abandonado, XJ5041, $570 \mathrm{~m}$, pie de muro, 18-IV-1991, A. Valdés \& R. Molina (ALBA 3157). $2^{\text {a }}$ cita provincial (Sánchez Gómez \& Alcaraz, 1993).

\section{Paspalum vaginatum $\mathrm{Swartz}$}

ALBACETE: Alcalá del Júcar, hacia Villavaliente, XJ3738, $630 \mathrm{~m}$, pastizal junto a una fuente, 26-VII-1992, R. Molina (ALBA 4889). $2^{\text {a }}$ cita provincial (Sánchez Gómez \& Alcaraz, 1993).

\section{* Phalaris canariensis L.}

ALBACETE: Villa de Ves, Barranco de Mingo,
XJ4839, $700 \mathrm{~m}$, borde de rambla algo nitrificada, 20-VI-1988, A. Valdés \& R. Molina (ALBA 2634).

\section{Polygonum equisetiforme $\mathrm{Sm}$.}

ALBACETE: Villa de Ves, Puntal Blanco, $\mathrm{XJ} 4842,500 \mathrm{~m}$, sotobosque de encinar con pino carrasco, 20-VI-1988, A. Valdés \& R. Molina (ALBA 3210). Villalgordo del Júcar, puente, WJ8151, $670 \mathrm{~m}$, maizales de ribera, 28-IX-1988, R. Molina (ALBA 3211). Segunda cita provincial ( Sánchez Gómez \& Alcaraz, 1093). También existe una cita genérica para la provincia en Castroviejo et al. (1990).

\section{Quercus x agrifolia Batt. in Batt. \& Trabut}

(Quercus rotundifolia $x$ Q. coccifera)

ALBACETE: Villa de Ves, Casa del Conde, XJ4840, $680 \mathrm{~m}$, encinar con Quercus coccifera, 6VI-1991, A. Valdés \& R. Molina (ALBA 3286). $2^{\text {a }}$ cita provincial (Sánchez Gómez \& Alcaraz, 1993).

Rubia peregrina L. subsp. Iongifolia (Poiret) O.Bolós

ALBACETE: Villa de Ves, entre Vado y El Molinar, XJ5139, $720 \mathrm{~m}$, madroñal en rambla con algo de humedad, 9-VI-1993, Alcaraz, Valdés \& Molina (ALBA 4928). Alcalá del Júcar, Tolosa, XJ4040, $540 \mathrm{~m}$, sauceda arbustiva, 9-VI-1993, Alcaraz, Valdés \& Molina (ALBA 4929). $2^{\text {a }}$ cita provincial (Sánchez Gómez \& Alcaraz, 1993).

\section{* Rumex roseus L.}

(R. tingitanus L.)

ALBACETE: Villalgordo del Júcar, hacia La Losa, WJ7952, $680 \mathrm{~m}$, tomillar algo nitrificado sobre suelos arenosos, 6-V-1992, R. Molina (ALBA 4767). La Roda, Puente del Carrasco, WJ8440, 680 m, pastizales sobre suelos arenosos, 16-IV-1992, R. Molina (ALBA 4922).

\section{* Saponaria officinalis L.}

CUENCA: El Picazo, hacia la presa, WJ7865, $750 \mathrm{~m}$, márgenes de regadíos de girasol, 11-VII1991, A. Valdés \& R. Molina (ALBA 3379).

\section{Sedum rubens $L$.}

ALBACETE: Villa de Ves, Casa Sandunga, XJ5140, $570 \mathrm{~m}$, espartal, 13-VII-1992, Alcaraz, Molina \& Valdés (ALBA 4697). $2^{\text {a }}$ cita provincial (Herranz \& Gómez Campo, 1986). 
* Silene nutans L. subsp. nutans

ALBACETE: Alcalá del Júcar, hacia Villavaliente, XJ3738, $630 \mathrm{~m}$, claros de encinar en ladera, 26-VII-1992, R. Molina (ALBA 5057).

* Spergularia boconnei (Scheele) Graebner in Asch. \& Graebner

ALBACETE: Villa de Ves, fuente, XJ5041, $600 \mathrm{~m}$, bordes arenosos de la carretera, 6-VI-1991, A. Valdés \& R. Molina (ALBA 3448).

Thymelaea tartonraira (L.) All. subsp. tartonraira ALBACETE: Jorquera, Los Alcozarejos, XJ2135, $610 \mathrm{~m}$, romeral sobre suelos calizomargosos, 13-V-1992, R. Molina (ALBA 4723). 2a cita provincial ( Sánchez Gómez \& Alcaraz, 1993).

\section{Valerianella carinata Loisel.}

ALBACETE: Villa de Ves, de Casa del Zurdo a Casa del Francés, XJ5439, 720 m, romerales, R. Molina \& J.L. González (ALBA 3491). 2 $2^{\text {a }}$ cita provincial (López Vélez, 1984 ).

Viola alba Besser subsp. dehnhardtii (Ten.) Nyman ALBACETE: Villa de Ves, Cristo de la Vida, XJ5041, $750 \mathrm{~m}$, ladera pedregosa con matorral de Cisto-Rosmarinetum, 21-IV-1990, R. Molina (ALBA 3524). Jorquera, hacia La Recueja, XJ2836, 540 m, olmeda-sauceda, 27-III-1990, R. Molina (ALBA 3525). Segunda cita provincial (Sánchez Gómez \& Alcaraz, 1993) y mención genérica de Castroviejo et al. (1993) para la provincia.

\section{BIBLIOGRAFÍA}

CASTROVIEJO, S., M. LAINZ, G. LÓPEZ GONZÁLEZ, P. MONTSERRAT, F. MUÑOZ GARMENDIA, J. PAIVA y L. VILLAR (eds.) 1986- Flora Iberica I. Serv. Publ. C.S.I.C. Madrid. 575 pp.

CASTROVIEJO, S., i . LAINZ, G. LÓPEZ GONZÁLEZ, P. MONTSERRAT, F. MUÑOZ GARMENDIA, J. PAIVA y L. VILLAR (eds.) 1990- Flora Iberica II. Serv. Publ. C.S.I.C. Madrid. 897 pp.

CASTROVIEJO, S., C. AEDO, S. CIRUJANO, M. LAINZ, P. MONTSERRAT, R. MORALES. F. MUÑOZ GARMENDIA, C. NAVARRO, J. PAIVA y C. SORIANO (eds.) -1993- Flora
Iberica III. Serv. Publ. C.S.I.C. Madrid. 730 pp. DANTÍN CERECEDA, J. -1911- Una excursión por los alrededores de El Salobral (Albacete). Bol. Soc. Esp. Hist. Nat. 11: 115-123.

HERRANZ SANZ, J.M. y C. GÓMEZ CAMPO 1986-Contribución al conocimiento de la flora y vegetación de la Comarca de Alcaraz (Albacete). Publ. Caja de Ahorros de Albacete. 279 pp.

HERRERO, A., A ESCUDERO y S. PAJARÓN 1994- Estudio florístico de la Sierra del Relumbrar. Inst. Est. Albacetenses. Serie I, $\mathrm{n}^{\circ}$ 80. Albacete. 219 pp.

LÓPEZ VÉLEZ, G. -1984-Contribución al conocimiento de la flora de los prados de la Cañada de Los Mojones, en el Calar del Mundo (Albacete). Al-Basit, año X, $\mathrm{n}^{\circ}$ 13: 175-184. Albacete.

LUCEÑO, M. -1994- monografía del género Carex en la Península Ibérica e Islas Baleares. Ruizia 14. 1-139.

MORODER, E. -1936- Relación de plantas herborizadas en Chinchilla (Albacete). Cavanillesia 8 (1-7): 63-64.

RIVAS MARTÍNEZ, S. -1966- Situación ecológica y fitosociológica del Lythrum flexuosum Lag. Bol. Soc. Esp. Hist. Nat. (Biol.) 64: 363-368.

SÁNCHEZ GÓMEZ, P. y ALCARAZ ARIZA, F. 1993- Flora, vegetación y paisaje vegetal de las Sierras de Segura Orientales. Inst. Est. Albacetenses. Serie I, n ${ }^{0}$ 69. Albacete. 459 pp. TUTIN, T.G., V.H. HEYWOOD, N.A. BURGES, D.M. MOORE, D.H. VALENTINE, S.M. WALTERS \& D.A. WEBB (eds.) -1964-1980Flora Europaea. Vols. I-V. Cambrigde University Press.

Aceptado para su publicación en Octubre 1997

Dirección de los autores. A. Valdés Franzi: Dpto. de Ciencia y Tecnología Agroforestal. E.U Magisterio. Plaza de la Universidad s/n. Universidad de Castilla-La Mancha. Albacete. R. Molina Cantos: Dpto. de Producción Vegetal. ETSI Agrónomos. Universidad de Castilla-La Mancha. Albacete. 